\title{
Should elderly men be screened for prostate cancer?
}
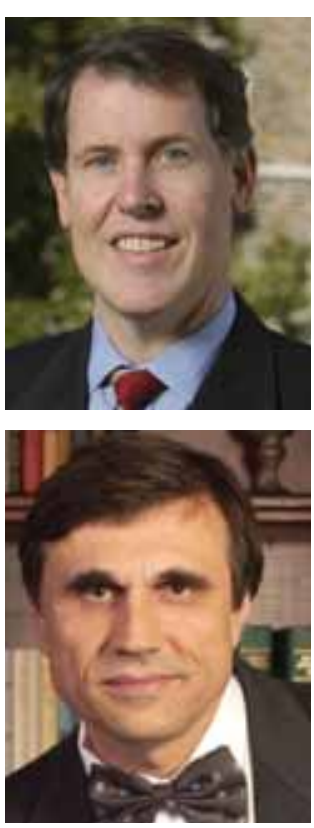

Judd W Moul ${ }^{1 \dagger} \&$ Vladimir Mouraviev²

${ }^{\dagger}$ Author for correspondence ${ }^{1} \mathrm{D}$ ivision of U rologic Surgery, D uke Prostate Center, $D$ epartment of Surgery, D ukeU niversity M edical Center, Box 3707, D urham, N C 27710, USA

Tel.: +1 919684 5057;

Fax: +1 919684 8794;

E-mail: judd.moul@duke.edu ${ }^{2}$ D ivision of U rologic Surgery, D epartment of Surgery, D uke U niversity M edical Center, Box 2804, D urham, NC 27710, USA

Tel.: +1 9196849055 ;

Fax: +1 919660 2258;

E-mail: moura002@

mc.duke.edu

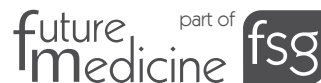

'...the use of PSAV in men having

multiple PSA measurements may

spare some men from unnec essary prostate biopsy.'

Prostate cancer ( $\mathrm{PCa}$ ) remains the most common, noncutaneous human malignancy with an estimated 186,320 new cases for 2008 in the USA. It is also the second most lethal tumor among men, with 28,660 expected deaths in 2008 [1].

Currently, the American Urological Association along with the Veterans Administration, American Academy of Family Physicians, the American Cancer Society, the American College of Physicians - American Society of Internal Medicine and the US Preventive Services Task Force recommend avoiding PCa screening in men with an estimated life expectancy of less than 10 years. However, in real practice, this approach remains extrem ely controversial. While some urologists advocate screening after the age of 70 years or recommend that biopsy for men with a prostate-specific antigen (PSA) level with a routine threshold $(<4 \mathrm{ng} / \mathrm{ml})$ is not worthwhile, other investigators search in-depth for specific features of development in this cohort of patients to adjust new data and PSA cutoffs according to concrete age. An even more contentious topic, assuming one is in favor of screening, is determining when it is reasonable and prudent to discontinue screening. At the same time, in the USA, predictions indicate that the population of people aged 65 years and older will rise to $21 \%$ by the year 2050 [101]. The number of people in the 65-84 years-old age group is expected to increase by over $100 \%$, and those in the 85 years and over age group will increase by approximately $400 \%$. This same trend is expected in other countries all over the world. Therefore, we need to pay much more attention to this quickly rising cohort of patients as part of a bigger healthcare problem, especially regarding the increased risk of age-related diseases. Furthermore, some patients with newly diagnosed advanced disease may require palliation if they are symptomatic, with a lifespan of less than 10 years. All of this, along with the announcement of an era for individual treatment approaches in oncology at the recent American Society of Clinical O ncology meeting, drives us to re-evaluate our traditional conservative attitude to this problem.

$\mathrm{H}$ offman presented an interesting point-topoint argument in 2006 in the Annals of Internal Medicine, which represented a viewpoint that advocated limited screening and strongly criticized screening men aged 75 years and older "because PCa is often indolent, and elderly men are unlikely to live long enough to benefit from treatment" [2]. He concluded that having a minimal treatment benefit in delayed fashion would meet a satiation of overscreening with potentially harmful consequences (such as unnecessary prostate biopsy) that could bring additional expenses to healthcare costs.

'...the discontinuation of sc reening in the elderly population is la rgely driven by decreased

life expectancy in a disease with a relatively long natural history.'

As a counterargument, Catalona et al. used the data from their research when lowering the PSA cutoff of $2.5 \mathrm{ng} / \mathrm{ml}$ for men aged 70 years and above and demonstrated that there was no significant difference in the proportion of men who underwent radical prostatectomy [3]. Therefore, elderly men can also be good candidates for radical treatment at a stage when cure is possible. By contrast, physicians do not wish to be in a position of treating clinically insignificant tumors, the treatment of which may cause greater morbidity than the disease itself. While $C$ atalona et al. suggest that screening is appropriate in selected patients of this age category [3], nowadays there are no clear guidelines to assist physicians in determining who is going to be screened or when to discontinue screening, based on age, in otherwise asymptomatic men. This editorial and other publications provoke much more research and literature in this field than that highlighted here. 
Age-adjusted prostate-specific antigen range

Until now, PSA screening along with the digital rectal examination remain the gold standard for the early detection of PC a despite the PSA's low specificity for the disease. In other words, because the PSA is not strictly specific for tissue and can be produced by normal and hyperplastic prostate tissue, a high false-positive rate frequently occurs. In fact, in men without $\mathrm{PCa}$, PSA levels increase with age, mainly because of increases in prostate volume secondary to age related benign prostatic hyperplasia. Therefore, age-specific PSA reference values were developed to account for age-related PSA changes.

Age-specific reference ranges attempt to decrease the likelihood of detecting clinically insignificant cancers in men above 70 years of age. The attempt to increase the PSA threshold up to $6.5 \mathrm{ng} / \mathrm{ml}$ in order to account for the increased prevalence of benign prostatic hypertrophy in this age group, while selecting appropriate candidates for prostate biopsy, has led to a missed diagnosis in $43 \%$ of all cases and in $47 \%$ of organ-confined cases [4].

'Despite continued controversy regarding the precise PSA cutoffs, there is little controversy that PSA screening guidelines should be sensitive to ethnic variations.'

U rologic oncologists may elect to use a lower PSA cutoff to better detect aggressive cancers that require treatment, or a higher PSA cutoff to avoid unnecessary biopsy in men with indolent cancers. For example, $\mathrm{N}$ adler et al. found a median PSA level of $2.5 \mathrm{ng} / \mathrm{ml}$ for men over the age of 60 years and suggested that cancer detection was similar to that found in younger men, with PSA levels between 2.6 and $4.0 \mathrm{ng} / \mathrm{ml}$ [5]. While clinically insignificant, they were often not detected by screening. Thus, the authors concluded that a lower PSA cutoff of $2.5 \mathrm{ng} / \mathrm{ml}$ could be considered, irrespective of age, in men who would be candidates for definitive treatment [5]. At the same time, they did not find any differences between PSA velocity (PSAV), tumor stage, $G$ leason grade and tumor volume in all age groups. U sing these ranges, the number of prostate biopsies can be reduced by $22 \%$ in men over the age of 70 years, while potentially increasing the sensitivity of the PSA test in younger men who are most likely to benefit from treatment if the cancer is detected early [5].
Although these studies suggested a certain threshold to discontinue screening, including in older men, the discontinuation of screening in the elderly population is largely driven by decreased life expectancy in a disease with a relatively long natural history. While we agree with screening-selected healthy men in their 70s, screening men in their 80 s and certainly $90 \mathrm{~s}$ is definitely not widely accepted.

\section{Other predictors in the elderly}

Another isoform of PSA for early detection, the PSAV, is the value of change in PSA levels with repeated measurements (two or more) over time (1.5-2 years). PSAV was introduced by $C$ arter et al. to improve PC a detection [6]. They determined that a PSAV of $0.75 \mathrm{ng} / \mathrm{ml} /$ year or greater ( $72 \%$ sensitivity and 95\% specificity) was strongly suggestive for PCa development, since the rate of change in PSA values with repeated measurements over time and the accurate use of PSAV for early detection requires the use of three PSA levels collected over approximately 1.5-2 years. Therefore, PSAV is more specific than routine PSA testing for the presence of PCa since only a few men $(\measuredangle \%)$ reach the PSAV cutoff required to trigger a prostate biopsy. The use of PSAV in the growing number of men with lengthy PSA histories obtained through systematic screening efforts in early detection may aid diagnosis and spare some men from unnecessary prostate biopsy. It was established that more than $95 \%$ of men without PCa will have a PSAV level of less than $0.75 \mathrm{ng} / \mathrm{ml} /$ year, whereas $72 \%$ of men with PC a will have a PSAV above this threshold. PSAV may be an additional tool indicating the development or presence of PCa because only a few men $(<5 \%)$ without the disease reach the PSAV cut-point to trigger a prostate biopsy. Furthermore, the use of PSAV in men having multiple PSA measurements may spare some men from unnecessary prostate biopsy. For instance, a recent study by $\mathrm{C}$ arter et al. demonstrated that PSAV may be considered as a reliable prognosticator of early PC a development when measured even 10-15 years before diagnosis, when most men had PSA levels in the normal range [7]. These changes in PSAV dynamics were associated with cancer-specific survival observed 25 years later. The disease-free survival rate was significantly higher $(92 \%)$ among men with a PSAV level of $0.35 \mathrm{ng} / \mathrm{ml} /$ year or less compared with $54 \%$ among men with a PSAV level above $0.35 \mathrm{ng} / \mathrm{ml} /$ year. Furthermore, men with a 
PSAV above $0.35 \mathrm{ng} / \mathrm{ml} /$ year had a significantly higher relative risk of death than men with a PSAV of $0.35 \mathrm{ng} / \mathrm{ml} /$ year or less.

Race may also be an important factor in setting a reference range. $M$ oul et al. demonstrated that African-American (AA) men, with or without PC a, have higher PSA levels [8]. Soon thereafter, $M$ organ et al. calculated age and raceadjusted reference ranges for PSA screening [9]. Despite continued controversy regarding the precise PSA cutoffs, there is little controversy that PSA screening guidelines should be sensitive to ethnic variations.

'...these results demonstrated ...

that there is no threshold of age at which to stop sc reening.'

M ouraviev et al. analyzed a large screening population of 4038 men who were over 70 years old, including 605 AA men and 3433 non-AA men from the Duke Prostate $C$ enter $O$ utcomes database [10]. The area under the curve (AUC) at the receiver operator characteristic (ROC) for PSA in AA men and non-AA men was 0.84 and 0.76 , respectively. For PSAV, the AUC was 0.71 versus 0.54 in $A A$ men and non-AA men, respectively. The largest relative sensitivity and specificity in AA men was achieved at the established PSA cut-point of $4.0 \mathrm{ng} / \mathrm{ml}-85$ and $71 \%$, respectively. The most favorable cut-point in non-AA men was $3.4 \mathrm{ng} / \mathrm{ml}$, resulting in a sensitivity and specificity of 72 and $73 \%$, respectively. The AUC of ROC curves within various age subgroups tended to be very stable regardless of how the ages were grouped. In a multivariate logistic-regression model, age, PSA and PSAV were significant predictors of PC $\mathrm{P}$ in the AA subset, while age and PSA were significant predictors in the non-AA subset. Finally, these results demonstrated, within various age and race subgroups, detection tends to be very stable from the early 70 s through to the mid $80 \mathrm{~s}$, and that there is no threshold of age at which to stop screening.

Moul et al. identified age-adjusted PSA and PSAV cut-points for PC a biopsy in a cohort of 33,643 men retrieved from the Duke Prostate Center database [11]. In 11,861 men, PCa prevalence was $273(8.0 \%), 659(14.9 \%)$ and 722 $(17.9 \%)$ in the groups of men aged $50-59$, 60-69 and 70 years or older, respectively. In PCa groups, the median PSA and PSAV levels in men aged $50-59$ years versus 70 years or above were 5.6 versus $8.1 \mathrm{ng} / \mathrm{ml}$ and 1.37 versus $1.89 \mathrm{ng} / \mathrm{ml} /$ year, respectively $(\varangle 0.0001)$. In men aged 50-59 years, the sensitivity and specificity were 82.1 and $80.7 \%$, respectively, at PSA levels $2.5 \mathrm{ng} / \mathrm{ml}$, and 84.3 and $72.4 \%$, respectively, at PSAV levels of $0.40 \mathrm{ng} / \mathrm{ml} /$ year. These levels were higher than those in men aged 70 years or above, at PSA $4.0 \mathrm{ng} / \mathrm{ml}$ or PSAV $0.75 \mathrm{ng} / \mathrm{ml} /$ year. Factors of age, sensitivity, specificity, positive predictive value and cancer prevalence are critical for obtaining the desired balance between PCa detection and negative biopsy.

Downside to increasing prostate-specific antigen screening beyond

guideline limits

A recent survey of general internists and family practitioners found that $81 \%$ implemented the PSA test into their health-maintenance exam for men aged 50 years and above [12]. Walter et al. demonstrated this extreme approach in 597,642 male veterans aged 70 years and above [13], a third (36\%) of them aged 85 years or above had received PSA screening and almost two-thirds $(64 \%)$ of men between 70 and 74 years of age had been screened. Furthermore, they revealed an increased amount of testing carried out in patients with serious, lifeshortening comorbidities. Therefore, these investigators found only a $7 \%$ difference between the testing rate in healthy elderly men versus those with poor health. Indeed, this study raised some concerns about whether PSA screening is still reasonable beyond the guideline limits with the growing national expenditures in this regard.

\section{Clinical behavior of prostate cancer}

in the elderly

Current literature supports that the clinical behavior of PCa may be more aggressive in elderly men. For instance, D 'Amico et al. analyzed the cause of death in 381 patients who underwent radiation therapy for clinically localized PC a [14]. They found that an age greater than or equal to 75 years at diagnosis is predictive of a shorter median time to death from PCa (6.3 vs 9.7 years; $p=0.002$ ) in high-risk patients. Patients with clinically localized, high-risk $\mathrm{PC}$ a who were diagnosed at the age of 73 years or above and were treated with radiation therapy had a worse prognosis compared with patients who were diagnosed at the age below 73 years, raising the possibility that a more aggressive $\mathrm{PCa}$ biology may develop during andropause. 
Our recent study investigating 14,601 patients with $\mathrm{PCa}$, including 4029 men who underwent radical prostatectomy with final pathology assessment, suggested that in two stratified age groups - below 70 years and 70 years or above - older men have poorer clinicopathological features and outcome [15], and it demonstrated that age has a marginal effect in predicting disease risk and outcome.

\section{Conclusion}

While many studies agree with the current official guidelines to avoid screening in men with a life expectancy of less than 10 years, other studies demonstrate a search for age-adjusted PSA levels and its isoforms, with an individual approach for the early detection of aggressive localized PC a that applys a more radical kind of treatment in selected healthy men in order to prolong their life span.

Financial \& competing interests disclosure Theauthorshaveno relevant affiliationsor financial involve ment with any organization or entity with a financial interest in or financial conflict with the subject matter or materials discussed in the manuscript. This includes employment, consultancies honoraria, stock ownership or options, expert testimony, grants or patents received or pending, or royalties.

$\mathrm{N}$ o writing assistance was utilized in the production of this manuscript.

\section{Bibliography}

1. Jemal $A$, Siegel $R$, Ward $E$ et al.: C ancer statistics, 2008. CA Cancer J. Clin. 58, 71-96 (2008).

2. H offman RM : Viewpoint: limiting prostate cancer screening. Ann. Intern. M ed. 144, 438-440 (2006).

3. Catalona WJ, Loeb S, H an M : V iewpoint: expanding prostate cancer screening. Ann. Intern. M ed. 144, 441-443 (2006).

4. O esterling JE, Jacobsen SJ, Chute C G et al.: Serum prostate-specific antigen in a community-based population of healthy men. Establishment of age-specific reference ranges. JAM A 270, 860-864 (1993).

5. N adler RB, Loeb S, Roehl KA, Antenor JA, Eggener S, C atalona W J: U se of $2.6 \mathrm{ng} / \mathrm{ml}$ prostate specific antigen prompt for biopsy in men older than 60 years. J. U rol. 174, 2154-2157; discussion 2157 (2005).

6. Carter H B, M orrell CH, Pearson JD et al.: Estimation of prostatic growth using serial prostate-specific antigen measurements in men with and without prostate disease. Cancer Res. 52, 3323-3328 (1992).
7. Carter H B, Kettermann A, Ferrucci $L$, Landis P, M etter EJ : Prostate-specific antigen velocity risk count assessment: a new concept for detection of lifethreatening prostate cancer during window of curability. U rology 70, 685-690 (2007).

8. M oul JW, Sesterhenn IA, Connelly RR et al.: Prostate-specific antigen values at the time of prostate cancer diagnosis in African-American men. JAM A 274, 1277-1281 (1995).

9. M organ TO, Jacobsen $S J, M \subset C$ arthy W F, Jacobson DJ, M CLeod D G, M oul JW: Age-specific reference ranges for prostatespecific antigen in black men. N. Engl. J. M ed. 335, 304-310 (1996).

10. M ouraviev V, Broadwater $G$, Sun $L$, M ayes JM, M oul JW, Polascik TJ: Can prostate-specific antigen and prostatespecific antigen velocity be used for prostate cancer screening in men older than 70 years? U rology 71, 1020-1023 (2008).

11. M oul JW, Sun L, H otaling JM et al.: Age adjusted prostate specific antigen and prostate specific antigen velocity cut points in prostate cancer screening. J. U rol. 177, 499-503; discussion 503-504 (2007).
12. Voss JD and Schectman JM : Prostate cancer screening practices and beliefs. J. Gen. Intern. M ed. 16, 831-837 (2001).

13. Walter $L C$, Bertenthal $D$, Lindquist $K$, Konety BR: PSA screening among elderly men with limited life expectancies. JAM A 296, 2336-2342 (2006).

14. D 'Amico AV, Cote K, Loffredo $M$, Renshaw AA, Chen M H : Advanced age at diagnosis is an independent predictor of time to death from prostate carcinoma for patients undergoing external beam radiation therapy for clinically localized prostate carcinoma. Cancer 97, 56-62 (2003).

15. Moul J, Sun L, Robertson $C$ et al.: Older men associated with advanced disease and poop outcome: an analysis of 1461 prostate cancer men in Duke Prostate Center. J. U rol. 179(Suppl.), 212 (2008).

Website

101. US C ensus Bureau, Population Division www.census.gov/population/www/projections /natproj.html 